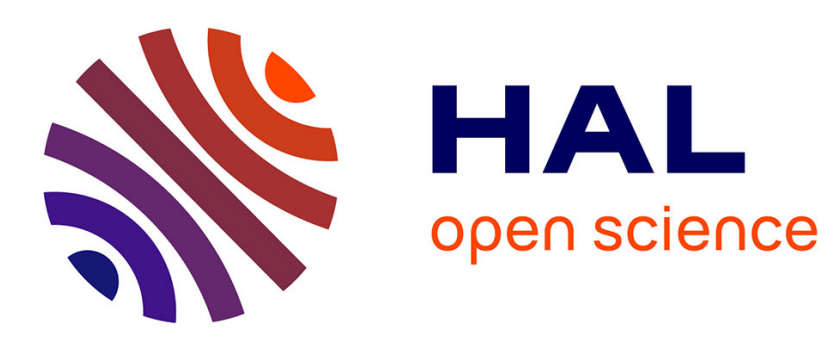

\title{
On the design of propeller hydrokinetic turbines: the effect of the number of blades
}

\author{
Antonio C. P. Brasil Junior, Rafael C. F. Mendes, Théo Wirrig, Ricardo \\ Noguera, Taygoara F. Oliveira
}

\section{To cite this version:}

Antonio C. P. Brasil Junior, Rafael C. F. Mendes, Théo Wirrig, Ricardo Noguera, Taygoara F. Oliveira. On the design of propeller hydrokinetic turbines: the effect of the number of blades. Journal of the Brazilian Society of Mechanical Sciences and Engineering, 2019, 41 (6), pp.1. 10.1007/s40430-0191753-4 . hal-02188625

\section{HAL Id: hal-02188625 \\ https://hal.science/hal-02188625}

Submitted on 18 Jul 2019

HAL is a multi-disciplinary open access archive for the deposit and dissemination of scientific research documents, whether they are published or not. The documents may come from teaching and research institutions in France or abroad, or from public or private research centers.
L'archive ouverte pluridisciplinaire HAL, est destinée au dépôt et à la diffusion de documents scientifiques de niveau recherche, publiés ou non, émanant des établissements d'enseignement et de recherche français ou étrangers, des laboratoires publics ou privés. 


\title{
On the design of propeller hydrokinetic turbines: the effect of the number of blades
}

\author{
Antonio C. P. Brasil Junior ${ }^{1} \cdot$ Rafael C. F. Mendes ${ }^{1} \cdot$ Théo Wirrig $^{2} \cdot$ Ricardo Noguera $^{2} \cdot$ Taygoara F. Oliveira $^{1}$
}

\begin{abstract}
A design study of propeller hydrokinetic turbines is explored in the present paper, where the optimized blade geometry is determined by the classical Glauert theory applicable to the design of axial flow turbines (hydrokinetic and wind turbines). The aim of the present study is to evaluate the optimized geometry for propeller hydrokinetic turbines, observing the effect of the number of blades in the runner design. The performance of runners with different number of blades is evaluated in a specific low-rotational-speed operating conditions, using blade element momentum theory (BEMT) simulations, confirmed by measurements in wind tunnel experiments for small-scale turbine models. The optimum design values of the power coefficient, in the operating tip speed ratio, for two-, three- and four-blade runners are pointed out, defining the best configuration for a propeller $10 \mathrm{~kW}$ hydrokinetic machine.
\end{abstract}

Keywords Hydrokinetic turbines · Propeller horizontal axis turbines · BEMT methods · Wind tunnel experiments · Glauert theory

\section{Introduction}

Hydrokinetic energy conversion devices have become an important choice of renewable energy resource in many worldwide regions. Applying different concepts of properly designed hydro turbines (e.g., Laws and Epps [1]), the kinetic energy of natural water streams (marine or river) can be converted into electricity for the supply of ocean, coastal or riverine societal needs, with low environmental impact and as a sustainable energy resource.

This technology has been reviewed by recent important number of works [1-4] in which the emergence of the

Taygoara F. Oliveira

taygoara@unb.br

Antonio C. P. Brasil Junior

brasiljr@unb.br

Ricardo Noguera

ricardo.noguera@ensam.eu

1 Laboratory of Energy and Environment, University of Brasilia, Brasília, DF 70910-900, Brazil

2 ENSAM - Ecole Nationale dArts et Métiers. Lab. DynFluid., 151, Bd. de l'Hôpital, 75013 Paris, France technology has been pointed out. One has reported several possibilities of technology applications (tidal and riverine), different machine design concepts and pre-commercial or $\mathrm{R} \& \mathrm{D}$ experiences, in the framework of the development of an important number of innovative hydrokinetic turbine converters.

Yuce and Muratoglu [4] had reported in 2015 about 300 relevant international project initiatives in hydrokinetic energy technology. Among them, $60 \%$ of the turbine designs are based in horizontal axis conception, mainly in the case of tidal applications. The hydrodynamical design methods for horizontal axis free flow hydrokinetic turbines are similar to those employed in wind turbines or to the propulsion propeller turbomachines. The design approaches can be adapted for the specific situations of hydrokinetic turbines, inheriting the important scientific rationale of the blade design of wind turbines $[5,6]$.

In general, the classical design approach for the optimized free flow runners is based on the Glauert blade element momentum theories [7]. It is a properly consolidated approach, presented in several wind turbine aerodynamics textbooks (e.g., Burton et. al.[8], Hansen [9] or Schaffarczyk [10]).

Important improvements in BEMT blade design approaches for wind turbines [11, 12], propellers [13] and 
hydrokinetic devices [14-18] have been explored in the literature, and some relevant ad hoc corrections associated with complex physical flow features (wake hydrodynamics, tip 3D effects, cavitation, etc.) have been proposed and integrated in BEMT approach. One has promoted robustness and accuracy in the methodology that may be associated with other advanced fluid mechanics methodologies such as CFD (computational fluid dynamics) and vortex methods, in a complementary framework for the blade hydrokinetic turbine design and its hydrodynamical assessment.

The design of the runners of horizontal axis hydrokinetic turbines allows an optimized geometry of their blade surfaces (here generated by the airfoil section piling in the blade axis), for a chosen number of blades. For larger machines (wind or tidal turbines), runners with three blades have been consolidated as the design selection, taking into account not only the hydrodynamics, but also others interdisciplinary aspects such as costs, weight and dynamics stability. Some small machines (medium and small size) have been proposed with a number of blades greater than three (see the review of Laws and Epps [1], for instance). The optimum number of blades for the hydrokinetic turbine runners is the basic question of the present paper.

The discussion of the number of blades in hydrokinetic or wind turbine is not too explored. Only a few references discussing the hydrodynamical effects on the choice of the number of blades are reported in the literature. Duquette et. al. [19] discussed the effect of the number of blades and the runner solidity for small wind turbines. This work concludes that the optimum design three-blade rotors produced an increase in the experimental power coefficient $-C p$ as solidity increased, with reduced tip speed ratio (TSR) at the optimum operating point. As blade number was increased at a constant solidity, the aerodynamic efficiency and power sharply decreased, contrary to the classical suggestion of Glauert [7] that pointed out the increase in $C p$ with the number of blades. In fact, the experimental work of Duquette does not explore properly the best optimum design for the given number of blades. Duquette et. al. [19] just change the number of blades in the rotor without making modification in the blade design. In the Glauert theory, the solidity has to be changed for the optimum design geometry, for each different number of blades.

In the work of Badshah et. al. [20], for tidal turbines with two-four blades, the experimental and BEMT results showed that the maximum value for nominal $C p$ remains the same to the different number of blades. Otherwise, a change in the higher TSR design point for rotors with more blades is observed and their torque is considerably higher for low TSR.

The wind tunnel results of Muhle et. al. [21] for wind turbines with two and three blades present the same behavior. The performances of both runners are quite the same, with a slight reduction in $C p$ for two-blade runner. The wake influence region did not change with the number of blades.

Previous results of BEMT and CFD computations of propeller hydrokinetic turbines with two-four blades were reported by Brasil Junior et. al. [22]. A small increase in $C P$ is observed for runners with the higher number of blades, changing the operation of low-TSR point. The wake influence region is not enlarged, but a considerable difference in the wake vortex pattern was observed, which influences the time-dependent flow behind the machine in different characteristics of frequencies.

The present paper explores the discussion of the effect of the number of blades in hydrokinetic turbines, using new wind tunnel experimental results, complemented to numerical simulations with BEMT approach. The revisiting of this problem, only in a hydrodynamical point of view, can promote the best design configuration of hydrokinetic turbines.

This work is organized as follows: In Sect. 2, the model of propeller hydrokinetic turbine is presented. Section 3 presents the design approach using the Glauert theory and BEMT formulations. In these sections, complete presentation of the classical method is developed in order to promote the completeness of the structure of the paper. In Sect. 4, the experimental methodology is presented, which is based on wind tunnel experiments. At last, Sect. 5 explores the results and their discussion.

\section{Hydrokinetic propeller turbine HK-10}

The present study is based on a propeller hydrokinetic turbines called HK-10 presented in Fig. 1. This machine, with $2 \mathrm{~m}$ diameter, has been designed to reach a rated power of $10 \mathrm{~kW}$ at a water stream velocity of $2.5 \mathrm{~m} / \mathrm{s}$. The Energy and Environmental Laboratory, at University of Brasilia, had held the hydrodynamic and mechanical design of the machine in collaboration with a group of R\&D partners. The main purpose of the proposed kinetic energy converter system is to obtain a sustainable technology to provide energy for small remote communities or for the recovery of the remaining energy potential at downstream of hydroelectric power plants. The modular and floating aspects of this system permit the adaptation of a group of turbines which work together, taking into account the local requirements of the power demand.

The hydrodynamical design parameters for this machine are presented in Table 1. The hydrodynamical design approach is applied considering those target variables. The analysis of the runner design with a different number of blades will be explored in the context of the optimized blade design approach. Two-, three- and four-blade runners will be considered in the present study (see Fig. 2) 
Table 1 Design parameters

\begin{tabular}{ll}
\hline Water flow velocity & $2.5 \mathrm{~m} / \mathrm{s}$ \\
Rotational speed & $35 \mathrm{rpm}$ \\
Rated power & $10 \mathrm{~kW}$ \\
Powertrain-generator efficiency & 0.9 \\
Power coefficient & $0.38-0.4$ \\
TSR $_{\text {op }}$ & $1.4-2.0$ \\
Number of blades & $2-4$ \\
\hline
\end{tabular}
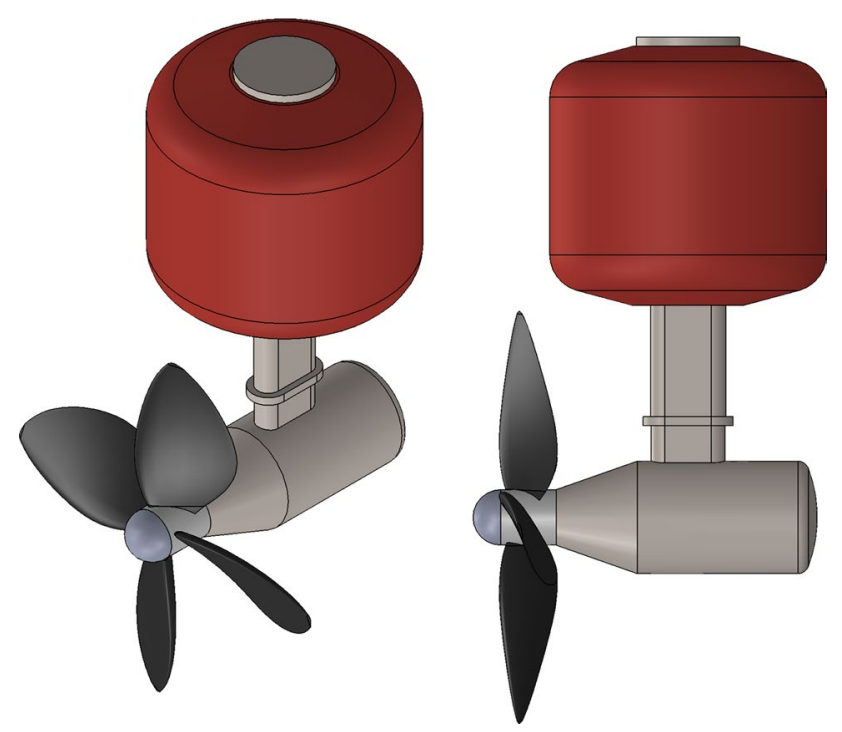

Fig. 1 HK10 propeller hydrokinetic turbine with four-blade runner (10 kW@2.5 m/s) and floater
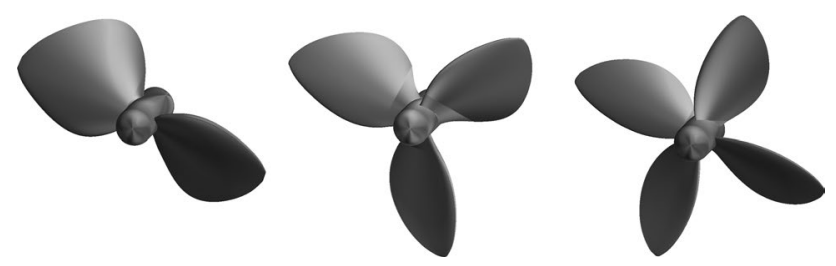

Fig. 2 Runners with a different number of blades - optimized blade design

\section{Blade element momentum theory}

\subsection{Runner kinematics}

The performance of a hydrokinetic turbine is expressed by the power coefficient, which is parameterized by its dependence to the tip speed ratio, denoted, respectively, by $C p$ and $\lambda$ classically defined as

$C p \equiv \frac{P}{1 / 2 \rho A V_{0}^{3}}$,

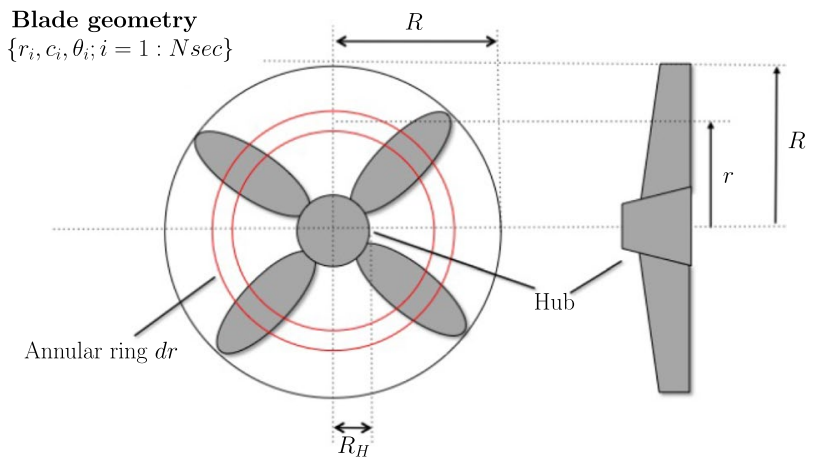

(a)

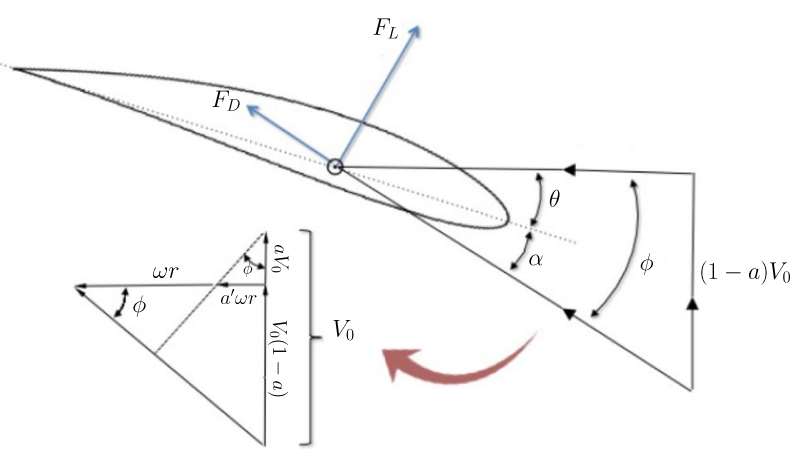

(b)

Fig. 3 Runner kinematics - velocity triangles in a blade section at radial position $r$

$\lambda \equiv \frac{\omega R}{V_{0}}$.

In those equations, $P, A$ and $R$ denote, respectively, the extract hydrodynamical power, area and radius of the runner and $\rho$ is the water density. The notation TSR (tip speed ratio) is also used alternatively for $\lambda$. The variables $V_{0}$ and $\omega$ are, respectively, the water flow velocity and the runner angular speed.

A parameterization of the axial velocity on the rotor cross-flow plane is written as

$V=(1-a) V_{0}$.

Additionally, on the outflow plane, the tangential velocity in the rotating wake in opposite to the rotor rotation can be expressed by a model of free vortex written as

$V_{\theta}=2 \omega r a^{\prime}$.

Equations 3 and 4 introduce the axial and tangential induction factors denoted by $a$ and $a^{\prime}$, respectively. Those variables are dependent on the radial position $r$.

In the rotor middle plane and at the radial position $r$, the velocity triangle on the blade section can be determined as illustrated in Fig. 3. The main velocity triangle is 
composed by the axial velocity $(1-a) V_{0}$ and the tangential component. The tangential component is equivalent to the blade velocity $\omega r$ added to the induced free vortex $a^{\prime} \omega r$, which turns in the opposite direction. Thus, the inflow angle $\phi$ can be computed by

$\tan \phi=\frac{(1-a) V_{0}}{\left(1+a^{\prime}\right) \omega r}=\frac{(1-a)}{\left(1+a^{\prime}\right)} \frac{1}{\lambda_{\mathrm{r}}}$,

with

$\lambda_{\mathrm{r}} \equiv \frac{\omega r}{V_{0}}$.

From the second small triangle, as illustrated in Fig. 3b, an additional relation yields

$\tan \phi=\frac{a^{\prime} \omega r}{V_{0} a}=\frac{a^{\prime}}{a} \lambda_{\mathrm{r}}$

From equations 5 and 7, a kinematic relationship between $a$ and $a^{\prime}$ can be obtained as

$a^{\prime}\left(1+a^{\prime}\right) \lambda_{\mathrm{r}}^{2}=a(1-a)$

or

$a^{\prime}=\frac{1}{2}\left(\sqrt{1+4 \lambda_{\mathrm{r}}^{-2} a(1-a)}-1\right)$.

\subsection{Axial and angular momentum balance}

The axial and angular momentum conservation principles can be formulated in the inflow and outflow sections through the runner. The integral 1D balance in an annular section of radius $d r$ gives the expressions for the thrust $d T_{1}$ and torque $d M_{1}$ differentials, which are written as

$d T_{1}=4 \pi r \rho V_{0}^{2} a(1-a) d r$,

and

$d M_{1}=4 \pi r^{3} \rho V_{0} \omega(1-a) a^{\prime} d r$.

The hydrodynamical extract power in $d r$ can be computed from Eq. 11 as

$d P=\omega d M=4 \pi r^{3} \rho V_{0} \omega^{2}(1-a) a^{\prime} d r$,

or in a dimensionless form

$d C p=\frac{8}{\lambda^{2}}(1-a) a^{\prime} \lambda_{\mathrm{r}}^{3} d \lambda_{r}$.

Therefore, the power coefficient for entire runner can be computed by integrating this equation in all its radius range given

$C p=\frac{8}{\lambda^{2}} \int_{0}^{\lambda}(1-a) a^{\prime} \lambda_{\mathrm{r}}^{3} \mathrm{~d} \lambda_{\mathrm{r}}$
In Eq. 14, a direct dependence of the runner performance to the radial distribution of the pair $\left\{a, a^{\prime}\right\}$ is figured out. Therefore, the optimization of the performance, which lies in the maximization of the $C p$, is directed related to finding the maximum value of the term $f\left(a, a^{\prime}\right) \equiv(1-a) a^{\prime}$.

Considering the relationship between $a^{\prime}$ and $a$, given in Eq. 9, the optimization problem related to the search of the maximum condition for $f\left(a, a^{\prime}\right)$ can be simplified to a onedimensional problem, only dependent on the variable $a$. It is the aim of the Glauert theory.

\subsection{Optimum flow conditions for runner design}

Considering the turbine nominal design variables given by $\left\{\omega, V_{0}, R\right\}$, which defines the values of $\lambda$, and for each radial position $\lambda_{\mathrm{r}}$. The maximum value of $d C p$, related to the maximum condition of $f\left(a, a^{\prime}\right)$, is simply obtained by the derivative of the function $f$ to $a$, i.e.,

$\frac{\mathrm{d} f}{\mathrm{~d} a}=(1-a) \frac{\mathrm{d} a^{\prime}}{\mathrm{d} a}-a^{\prime}=0$.

Using this equation with the derivative of Eq. 9, a cubic polynomial expression can be obtained as

$16 a^{3}-12 a^{2}+3\left(3-\lambda_{\mathrm{r}}^{2}\right) a-\left(1-\lambda_{\mathrm{r}}^{2}\right)=0$.

Therefore, for a blade radial position expressed by $\lambda_{\mathrm{r}}$, the unique real root greater than zero of Eq. 16 defines the optimum flow condition for the runner. The equivalent value for the radial induction factor can also be obtained as

$a^{\prime}=\frac{(1-3 a)}{(4 a-1)}$.

Using this reasoning, for a given value of $\lambda_{\mathrm{r}}$, equations 16 and 17 can be used to obtain the values of $\left\{a, a^{\prime}\right\}$. Thus, Eq. 5 can be employed complementary to obtain the value of the inflow angle $\phi$.

The analytical solution to find the root for the Glauert polynomial in Eq. 16 can be simply implemented using (e.g., [23])

$a=\frac{1}{2}\left[1-\lambda_{\mathrm{r}}\left(\cos \theta^{+}-\cos \theta^{-}\right)\right]$,

with

$\lambda_{\mathrm{r}}=\sqrt{\left(1+\lambda_{\mathrm{r}}\right)}$

and

$\theta^{ \pm}=\frac{1}{3} \cos ^{-1}\left( \pm \lambda_{\mathrm{r}}^{-1}\right)$.

Considering the Glauert approach to determining optimum flow conditions for the runner, Algorithm A1 can be proposed. 


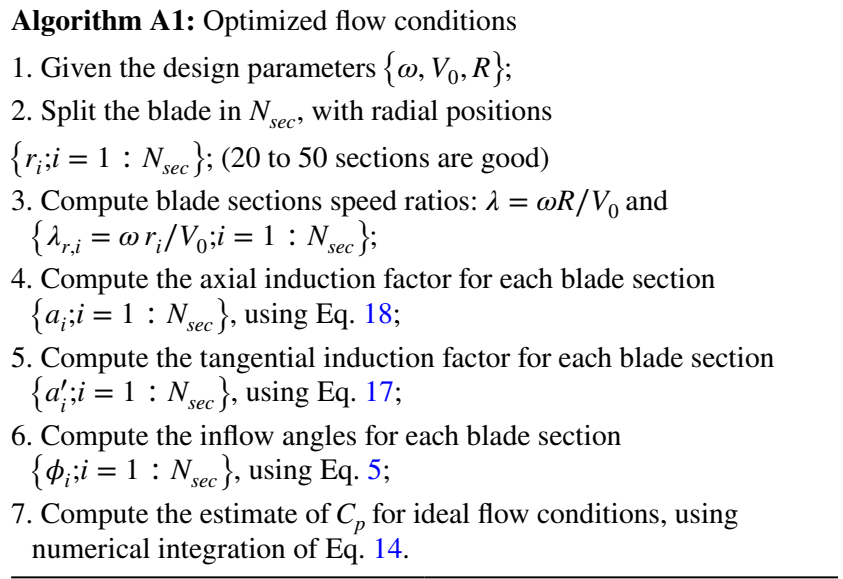

\subsection{Hydrodynamical forces on blade sections}

The second component of BEMT is the estimate of the hydrodynamical forces on each blade section. It is obtained computing the lift and drag forces on airfoil sections which composes the blades, using the standard airfoil theory and data.

In the BEMT, the computation of the hydrodynamic forces in a blade element $d r$ is obtained by the properly vectorial projection of the drag and lift on the axial and tangential directions. Thus, for a rotor with $N_{b}$ blades, the thrust and the moment on blade sections can be computed by

$d T_{2}=\frac{1}{2} \rho N_{b} C \frac{V_{0}^{2}(1-a)^{2}}{\sin ^{2} \phi} C_{n} d r$

and

$d M_{2}=\frac{1}{2} \rho N_{b} C \frac{V_{0}(1-a) \omega r\left(1+a^{\prime}\right)}{\sin \phi \cos \phi} C_{t} r d r$.

In those equations, $C$ denotes the chord length on the blade section and $C_{n}$ and $C_{t}$ are defined by

$C_{n}=C_{L} \cos \phi+C_{D} \sin \phi$

and

$C_{t}=C_{L} \sin \phi-C_{D} \cos \phi$.

At this moment, the lift and drag coefficients of the foil sections are introduced, where $C_{L}=C_{L}(\alpha)$ and $C_{D}=C_{D}(\alpha)$ are available from the data for standard airfoils. Here, $\alpha$ denotes the angle of attack of the flow relative on the blade section (see Fig. 3b). The NACA four-digit foils are used in the present work, and their polar curves are obtained from the open-source code XFOIL [24].

In order to compute hydrodynamical losses in the framework of complex fluid flow phenomena in the rotating blades (e.g., tip and hub losses), a $F\left(r, N_{b}\right)$ correction function is introduced. In the present work, Prandtl's tip and hub losses corrections are considered, changing equations 10 and 11 to

$d T_{1}=4 \pi r \rho V_{0}^{2} a(1-a) F\left(r, N_{b}\right) d r$

and

$d M_{1}=4 \pi r^{3} \rho V_{0} \omega(1-a) a^{\prime} F\left(r, N_{b}\right) d r$,

with

$F_{\text {tip }}=\frac{2}{\pi} \cos ^{-1}\left[\exp \left(-\frac{N_{b}}{2} \frac{(R-r)}{r \sin \phi}\right)\right]$

and

$F_{\text {hub }}=\frac{2}{\pi} \cos ^{-1}\left[\exp \left(-\frac{N_{b}}{2} \frac{\left(r-R_{\text {hub }}\right)}{r \sin \phi}\right)\right]$.

Using those two corrections functions, only acting, respectively, on the blade sections close to the tip and close to the hub, one can write

$F\left(r, N_{b}\right)=F_{\text {tip }} F_{\text {hub }}$.

Remark 1 For the blade design phase, an optimum angle of attack has to be selected in the airfoil data. This angle, denoted by $\alpha_{\text {op}}$, is obtained from the airfoil polar data where the ratio $C_{L} / C_{D}$ is maximum.

Remark 2 An estimate of the chord Reynolds number has to be considered, in order to use XFOIL. It can be obtained iteratively from all steps of geometry design procedures. In general, the airfoil polars are not too sensitive to the close values of Reynolds number and a first estimate of Reynolds can be considered from the values of the chord dimensions of existing similar machines.

Remark 3 For runners with high solidity, defined here by

$\sigma_{r} \equiv \frac{N_{b} C}{2 \pi n r}$

a correction considering the effects of cascade flow has to be taken into account. It was introduced in the design of propeller hydrokinetic turbines, only with a greater number of blades $N_{b} \geq 5$ and $\sigma_{r}^{-1}<3$, when the effect of the proximity of the blades has to be included in the formulation (see [22] or [25]).

\subsection{Optimized blade geometry}

From a design point of view, Algorithm A1 provides the values of the induction factors ( $a$ and $a^{\prime}$ ) and the inflow angle $\phi$, which establishes the optimized flow conditions for each radial position. The runner design has to be complemented with the definition of the blade shape, expressed 
by the chord $C$ and twist angle $\theta$ for different sections distributed through its length, i.e., $\left\{C_{i}, \theta_{i} ; i=1: N_{\text {sec }}\right\}$.

From equations 25 and 21 , with $d T_{1}=d T_{2}$, a closed expression for the chord length distribution $C(r)$ can be obtained

$\frac{C(r)}{R}=\frac{8 \pi}{N_{b}} \frac{a}{(1-a)} F\left(r, N_{b}\right) \frac{\sin ^{2} \phi}{C_{n}} \frac{r}{R}$.

At last, the blade twist angle can be determined from the optimum foil angle of attack (see Fig. 3b), using

$\theta=\phi-\alpha_{\mathrm{op}}$

Therefore, the algorithm for the blade design can be proposed

\footnotetext{
Algorithm A2: Optimized blade geometry

1. Given the design parameters $\left\{\omega, V_{0}, R, N_{b}\right\}$;

2. Given optimum flow conditions from Algorithm A1 $\left\{a_{i}, a_{i}^{\prime}, \phi_{i} ; i=1: N_{s e c}\right\}$

3. Using XFOIL, obtain $\alpha_{\text {op }}$ from maximum condition of $C_{L} / C_{D}$, and $C_{L}\left(\alpha_{\mathrm{op}}\right)$ and $C_{D}\left(\alpha_{\mathrm{op}}\right)$;

4. Compute chord lengths using Eq. $31\left\{C_{i} ; i=1: N_{\text {sec }}\right\}$;

5. Compute blade twist angle using Eq. $32\left\{\theta_{i} ; i=1: N_{\text {sec }}\right\}$;
}

\subsection{Performance analysis using BEMT}

The formulation presented in the two previous subsections was implemented by means of a MATLAB $\odot$ code using Algorithms A1 and A2. It permits the complete shape design of the blade geometry for axial flow hydrokinetic turbines. The runners designed by this approach will be evaluated with two different methodologies: numerical simulations using BEMT and small-scale wind tunnel experiments.

The BEMT formulation can also be used for performance analysis, permitting numerical simulations of the behavior of the turbine performance, in a large range of TSR. The second approach used in the present paper will explore the experimental measurements in wind tunnel experiments. Hence, a good validation of the estimates of machine performance can be assessed, obtaining the performance curves $(C p \times \mathrm{TSR})$, torque and thrust characteristics.

In performance simulations, two additional equations can be obtained using $d T_{1}=d T_{2}$ and $d M_{1}=d M_{2}$. Thus, from Eq. 25 equal to Eq. 21 and Eq. 26 equal to Eq. 22 the following relations can be obtained

$\frac{a}{1-a}=\frac{\sigma_{r} C_{n}}{4 F \sin ^{2} \phi}$

and

$\frac{a^{\prime}}{1+a^{\prime}}=\frac{\sigma_{r} C_{t}}{4 F \sin \phi \cos \phi}$.
Or alternatively

$a=\left(\frac{4 F \sin ^{2} \phi}{\sigma_{r} C_{n}}+1\right)^{-1}$

and

$a^{\prime}=\left(\frac{4 F \sin \phi \cos \phi}{\sigma_{r} C_{t}}-1\right)^{-1}$.

An iterative approach can be employed to compute the values of $\left\{a, a^{\prime}\right\}$, using the nonlinear problem formulated by Eq. 35 and Eq. 36, for all blade radial positions and for any machine operating point, given by $\lambda$ (for details, see Hansen [9], for instance).

Therefore, the power coefficient can be computed by the numerical integration of Eq. 14 , correcting the losses formulating by Eq. 26, i.e.,

$C p=\frac{8}{\lambda^{2}} \int_{\lambda_{h}}^{\lambda} F\left(r, N_{b}\right)(1-a) a^{\prime} \lambda_{\mathrm{r}}^{3} \mathrm{~d} \lambda_{\mathrm{r}}$

In this equation, $\lambda_{h} \equiv \omega R_{h} / V_{0}$.

The method has to be complemented by an important condition when the values of the axial induction factors surpass 0.4. In this situation, the formulation of the BEMT is not valid due to the complex and important $3 \mathrm{D}$ effects in the runner flow (Liu and Janajreh [11], for instance). In this situation, if $C_{T} \geq 0.96$, an empirical condition for the axial induction factor has to be formulated as

$a=\frac{\left(18 F-20-3 \sqrt{C_{T}(50-36 F)+12 F(3 F-4)}\right)}{(36 F-50)}$.

This correction was properly formulated by Buhl [12], using the basic ideas of Glauert and an alternative curve fit of experimental observations for the thrust coefficient, $C_{T}$, which is defined here by

$C_{T} \equiv \frac{T}{1 / 2 \rho A V_{0}^{2}}$,

and can be computed in the BEMT approach, for a specific radial section using

$d C_{T}=4 F a(1-a)$.

In the present paper, for the BEMT computations, the opensource numerical tool QBLADE is employed (Marten et. al. [26]). In this code, the BEMT equations are implemented in a robust and friendly simulation platform, equipped with a XFOIL interface for the polar curve computations. This numerical tool permits the performance analysis in the entire range of TSR, considering the corrections for tip and hub 
losses, as well as the Buhl-Glauert correction for high values of axial induction factor. All designed blade geometries have been considered for the BEMT simulations using properly QBLADE setting parameters.

\subsection{Hydrodynamical losses}

The hydrodynamical process to extract energy from the flow on the ideal condition is expressed by the dimensionless integral in Eq. 14. The maximum amount that it could have occurred is given by the Betz limit (59.26\%). Glauert [7] has shown that for low-TSR regimes, this limit is more restrictive, due to the rotational effects in the wake flow.

Considering the amount of energy which can be converted mechanically by the runner, one part of its power is consumed by the drag on the turbine blades. Another part is consumed by other complex flow phenomena associated with the $3 \mathrm{D}$ effects in the flow through the runner (mainly in tip and hub regions).

An approximated formulation can be obtained to express mathematically those effects. Firstly, Eq. 24 is simplified neglecting the drag, i.e.,

$C_{t} \approx C_{L} \sin \phi$

Substituting this approximation in Eq. 34, the following relation can be obtained

$\left(1+a^{\prime}\right) \approx a^{\prime} \frac{8 \pi r F}{N_{b} C} \frac{\cos \phi}{C_{L}}$.

Substituting it in Eq. 22 and integrating on the entire blade, the following expression can be obtained

$C p \approx \frac{8}{\lambda^{2}} \int_{\lambda_{h}}^{\lambda} F\left(r, N_{b}\right)(1-a) a^{\prime}\left(1-\frac{C_{D}}{C_{L}} \cot \phi\right) \lambda_{\mathrm{r}}^{3} \mathrm{~d} \lambda_{\mathrm{r}}$.

The different components in this equation quantify the relative losses compared to the ideal flow (given by Eq. 14)

- Drag losses Given by $\left(1-\frac{C_{D}}{C_{L}} \cot \phi\right)$, it explicit depends on $C_{D} / C_{L}$ ratio.

- Tip and hub losses Estimated by the Prandtl term $F\left(r, N_{b}\right)$, it has to be integrated in entire blade length.

Some remarks can be pointed out:

Remark 4 The design procedure that has selected the best foil standard geometries and $\alpha_{\mathrm{op}}$, considering a maximum value of $C_{L} / C_{D}$, allows the better choice to minimize the drag losses, as is shown in Eq. 43.

Remark 5 The term $F\left(r, N_{b}\right)$ is the only part in the formulation which accounts for the number of blades. This term tends to one when $N_{b}$ goes to a higher value (ideal condition related to an infinite number of blades).

The formulation presented in this section allows a complete theoretical modeling approach to design and assess the energy conversion on the hydrokinetic turbines. Simply numerical tools can be developed, in the framework of the evaluation of the number of blades effects in these machines performance, which is closely related to the influence of the correction factor $F(r, N b)$.

\section{Experimental setup and procedures}

In this section, the experimental methodology is presented, which is based on small-scale tests in the wind tunnel facility. The proposed approach was developed as an alternative of experimental performance evaluation of hydrokinetic turbines, generally based on experiments in flumes (e.g., [27]).

Wind tunnel experiments, complemented by a modelto-prototype transposition approach, are employed in the present study. The results for the small-scale experiments in airflow can be extrapolated to the water flow on the prototype scale in water. It is obtained by a calibrated methodology using also BEMT arguments. It has been validated by means of CFD numerical simulations in both scaling levels [28].

\subsection{Wind tunnel facilities and turbine small-scale models}

The experiments were performed in the open-loop wind tunnel of the Laboratory of Energy and Environment of University of Brasilia. With a test section of $1.2 \mathrm{~m} \times 1.2 \mathrm{~m}$ with $2 \mathrm{~m}$ long and a contraction ratio of $8: 1$, the $10 \mathrm{~kW}$ wind tunnel can reach a controlled air speed up to $20 \mathrm{~m} / \mathrm{s}$, with turbulence intensity less than $0.2 \%$. Figure 4 presents a general overview of the test facility.

Inside the wind tunnel, a pitot tube is used to measure the free-stream speed. The air temperature and relative humidity are monitored using calibrated electronic sensors. The experimental uncertainty of velocity measurements is \pm 0.1 $\mathrm{m} / \mathrm{s}$.

A small-scale one-tenth turbine model was installed inside the wind tunnel test section as shown in Fig. 5. The different runner models are shown in Fig. 6. Those runners are manufactured in an ABL 3D printer system and finished by epoxy paint. Its dimensions are checked after the manufacturing process ensuring the variations between the $3 \mathrm{D}$ CAD design and the final model keep under $0.1 \mathrm{~mm}$. 
Fig. 4 Experimental setup

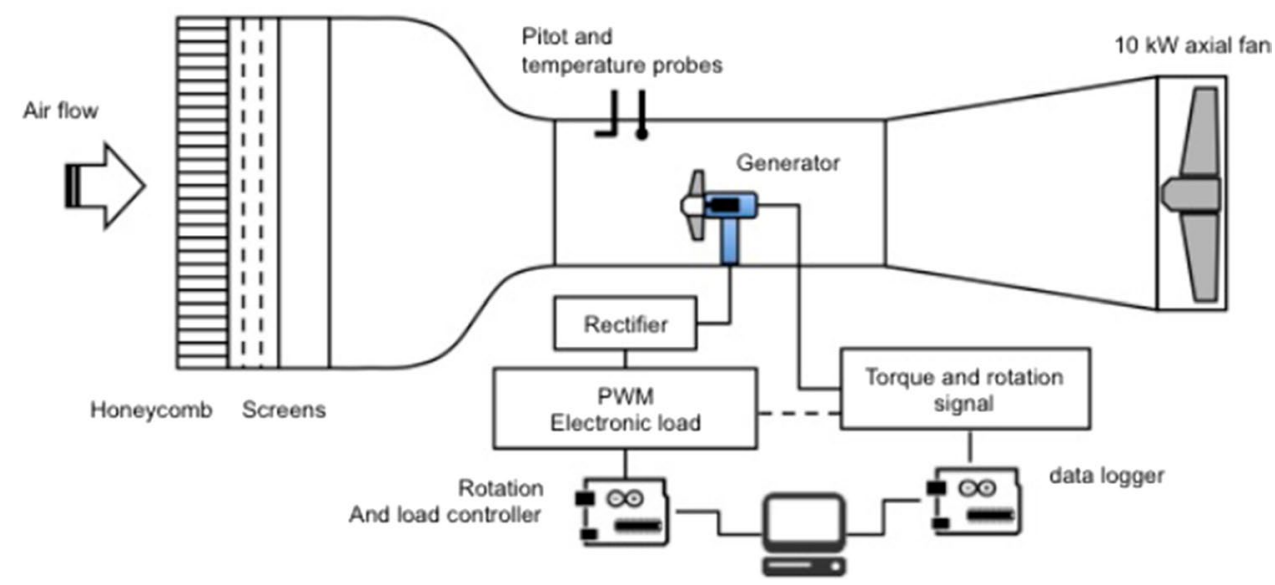

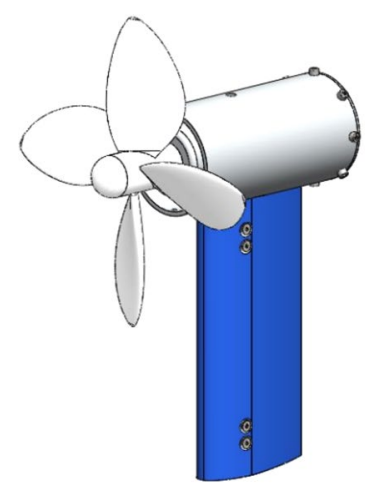

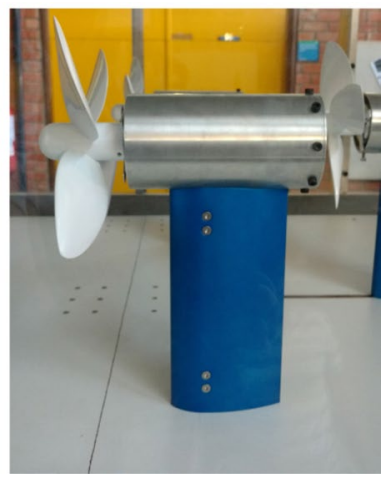

Fig. 5 Small-scale turbine inside wind tunnel

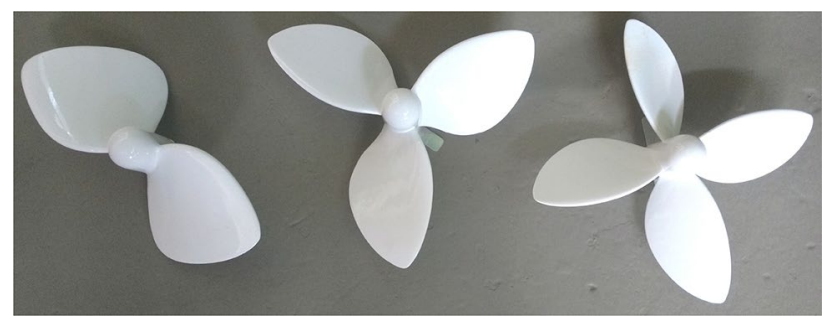

Fig. 6 Small-scale runners with two, three and four blades

\subsection{Control, torque and rotation measurements}

A small brushless permanent magnet generator is used as a brake inside the nacelle of the turbine model. It maintains the turbine rotor operation at a constant rotational speed. The control was based on an electronic-resistive load circuit, as illustrated in Fig. 7, where it is possible to set the rotational speed changing the switch time by means of a PWM-PID controller. The closed-loop control system achieves a stable rotation for given wind speed, using different settings for the load levels on the generator. It is completely controlled by an Arduino@ code.

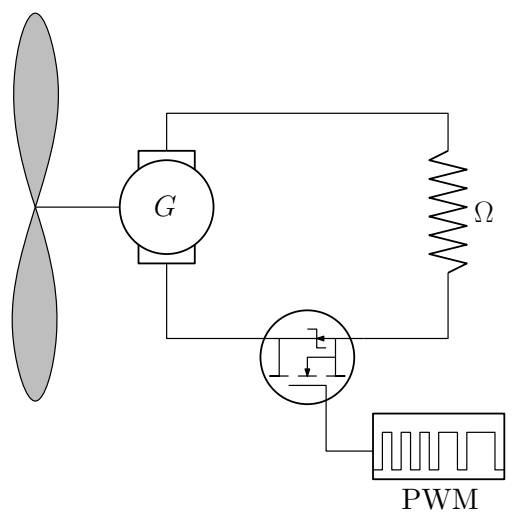

Fig. 7 Control system. G: turbine generator. $\Omega$ : electric load

The torque, $T$, was measured using a torsional load cell, as illustrated in Fig. 8. The turbine model was constructed to permit only one inertia referential support for the generator. By this way, all the generator's torque is transmitted directly to the torsional cylinder, where four strain gauges, of $120 \mathrm{ohms}$ each, were fixed, integrating a complete Wheatstone bridge. Any torque variation in generator shaft causes a deformation in the load cell and in the strain gauges, producing an unbalancing in the bridge. The voltage drops are related to torques acting in the shaft by a static calibration procedure. As the rotor is connected to the generator, the assembly allows to measure rotor torque. The torque meter system has an accuracy of $\pm 10^{-4} \mathrm{~N} \cdot \mathrm{m}$.

The operation of the turbine model maintains the rotational speed, $N$, constant in a fixed value within $\pm 10 \mathrm{rpm}$ variations in a range of 300-2500 rpm (measured by an optical infrared rotation sensor, with accuracy of $0.1 \mathrm{rpm}$ ).

Therefore, the power converted in the turbine is calculated by

$P=\omega T, \omega=\frac{N \pi}{30}$. 


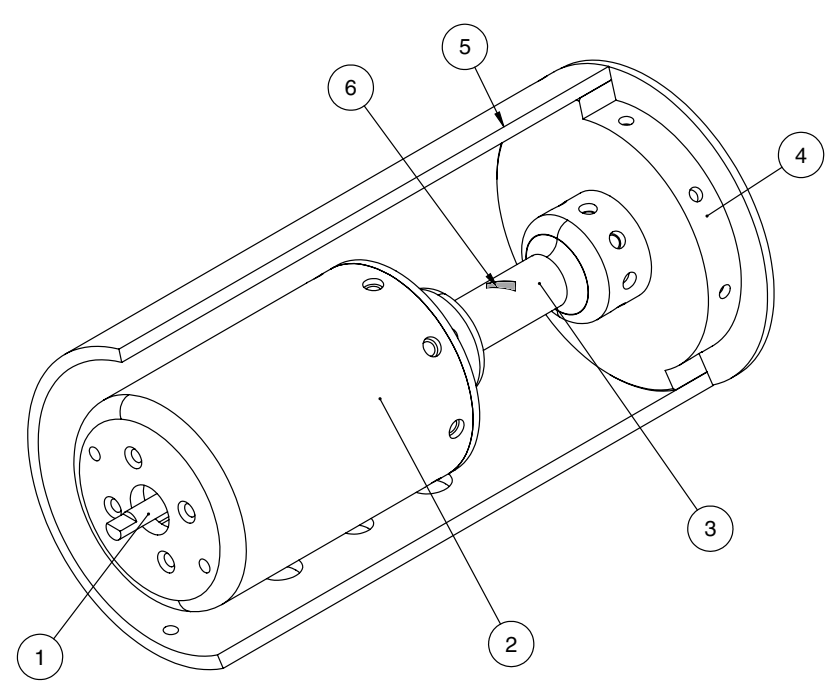

Fig. 8 Torsional load cell parts. 1: generator shaft, 2: generator support, 3: torsional cylinder, 4: fixation plate, 5: nacelle shell, 6: strain gauge

\subsection{Experimental procedure}

The experimental runs aim to produce curves of power coefficient as a function of the tip speed ratio, $C p \times \mathrm{TSR}$. In this work, TSR variations are generated by keeping the freestream velocity $V_{0}$ constant and varying the rotation speed, $\omega$, in such way that the Reynolds number based on the averaged chord length is kept constant.

For performance evaluation, the experimental runs start by setting the free flow speed at a fixed value $(12 \mathrm{~m} / \mathrm{s}$ is used). The PWM control system was programmed to sweep a pre-settled range of runner rotational speed (300-2500 rpm), by changing automatically the electrical load. The experimental control system varies the rotation for entire range of the turbine TSR $(0.25-2.5)$. The data logger registers the turbine torque for each situation. By this way, an entire $C p \times$ TSR curve is obtained. All experimental procedure is completely controlled by a MATLAB $\odot$ interface and data logging code. All results were obtained for the three different configurations of runners, and the error report is presented in "Appendix A."

\section{Results and discussion}

\subsection{Optimized blade geometry}

The design methodology, presented in Sect. 3 and summarized in Algorithms A1 and A2, was implemented in a MATLAB $\odot$ code. It allows us to compute the blade shape for a given set of input parameters $\left\{V_{0}, \omega, R, N_{b}\right\}$. The computation of the blade radius is performed considering the

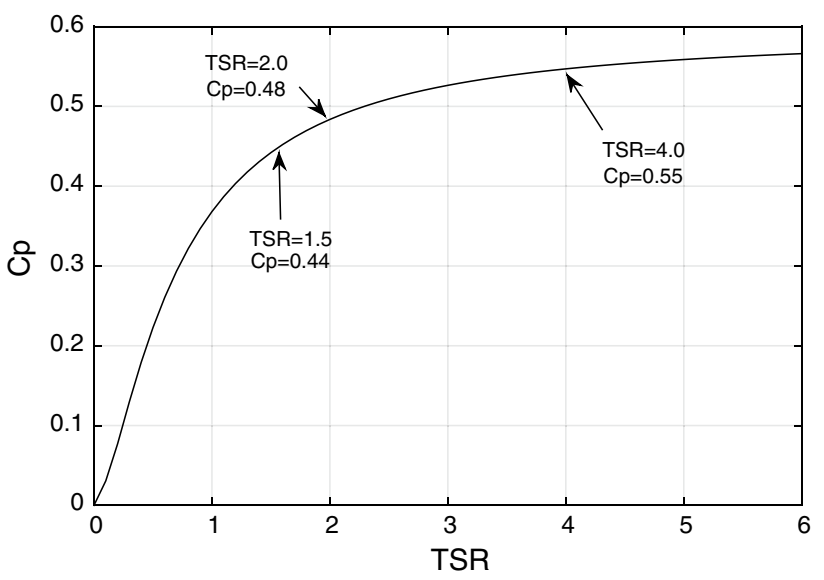

Fig. 9 Optimum Glauert power coefficient-infinite number of blades

design power-extract target in the machine requirements. An iterative approach can be used, computing successively the power coefficient and the nominal tip speed ratio, up to the convergence to the selected value of the power.

In the present paper, the blade design employs the foil NACA4415, with its polar curves obtained from the software XFOIL, with a typical Reynolds number around 2.0e+06. Only the value of $\alpha_{\mathrm{op}}$ and the evaluation of $C_{L}\left(\alpha_{\mathrm{op}}\right)$ and $C_{D}\left(\alpha_{\mathrm{op}}\right)$ are needed as input data in Algorithm A2.

In Fig. 9, the curve of $C p \times$ TSR for an infinite number of blades and for ideal flow conditions is illustrated, highlighting the values of three different design conditions for runner machines in terms of TSR (1.5, 2.0 and 4.0). Those choices represent different specific characteristics for slow and fast rotational speed machines analyzed here. The values of $C p$ in this plot do not take into account realistically the hydrodynamical losses through the runner. It is only computed from the optimum flow conditions of $\left\{a, a^{\prime}\right\}$, issued from Algorithm A1 and by means of the integral in Eq. 14. It can be considered as a limit curve for the behavior of the designed runner, which replaces the Betz limit, in particular for low-TSR regimes (classical Glauert curve).

In Fig. 10, losses are taken into account as formulated in Eq. 43. The flow continues to be ideal in the sense of the Glauert polynomial (i.e., the best conditions for $a$ and $a^{\prime}$ ). Otherwise, the terms of drag losses and tip/hub losses are considered. This last term influences the reduction in $C p$ values for runners with a low number of blades, corroborating with many theoretical, numerical and experimental observations (e.g., [20, 22]).

In Table 2, the values of $C p$ in different TSR conditions are presented. Those values consider the losses in a optimum flow condition for $\left\{a, a^{\prime}\right\}$ using Eq. 43.

For low-speed machines, an important difference in the $C p$ levels is observed for the different number of blades. In the runner of two blades, the lowest level of performance 


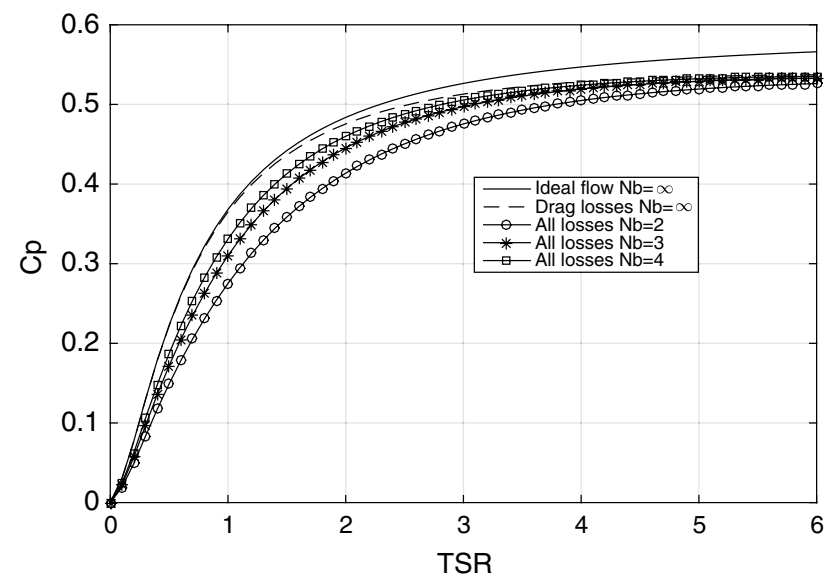

Fig. 10 Optimum Glauert power coefficient—considering losses

Table $2 C p$ for ideal flow conditions at selected TSR points (with drag and tip/hub losses)

\begin{tabular}{llll}
\hline Number of Blades & TSR $=1.5$ & TSR $=2.0$ & TSR=4.0 \\
\hline 2 & 0.36 & 0.41 & 0.50 \\
3 & 0.39 & 0.45 & 0.51 \\
4 & 0.41 & 0.46 & 0.52 \\
$\infty$ & 0.44 & 0.48 & 0.53 \\
\hline
\end{tabular}

induces the use of design with runners with three or more blades. This difference is not too important for faster (or larger) machines, with greater TSRs. For this condition, runners with two or three blades can be designed, as suggested by Muhle et. al. [21] for wind turbines, without a relevant reduction in the extract power performance.

In Fig. 11, a general overview of the optimized shapes for different design TSRs and number of blades is illustrated. This result shows that thinner shapes are obtained for faster machines.

The optimized runner shapes, for the design conditions of HK-10 machine, as presented in Table 1, are close to $\mathrm{TSR}=1.5$. The 3D runners for this condition are presented in Fig. 2. It is a slow machine, in which the blades are wider and the maximum power coefficient is limited around 0.4. Unfortunately, other design requirements for that machinerelated to the mechanical and electrical components in the powertrain-restrain the rotational speed of the turbine at a lower level. It has a constraint on the power coefficient up to 0.4 ; otherwise, it gives reliability for a machine operating in lower rotation speeds.

The volume of the designed runners can be calculated from an integral of chord length distribution, using the standard foil geometry. Its values (expressed in dimensionless form) for a different number of blades up to 5 are shown in Fig. 12. It can be observed a reduction in the volume of

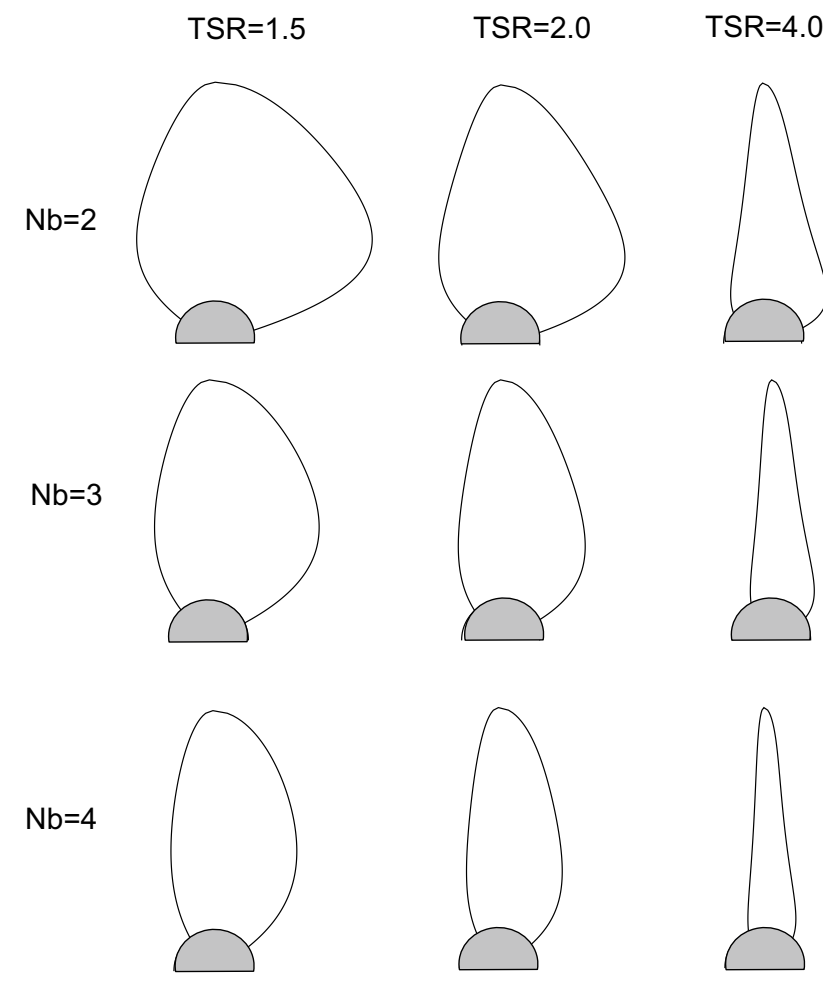

Fig. 11 Optimized blade geometries

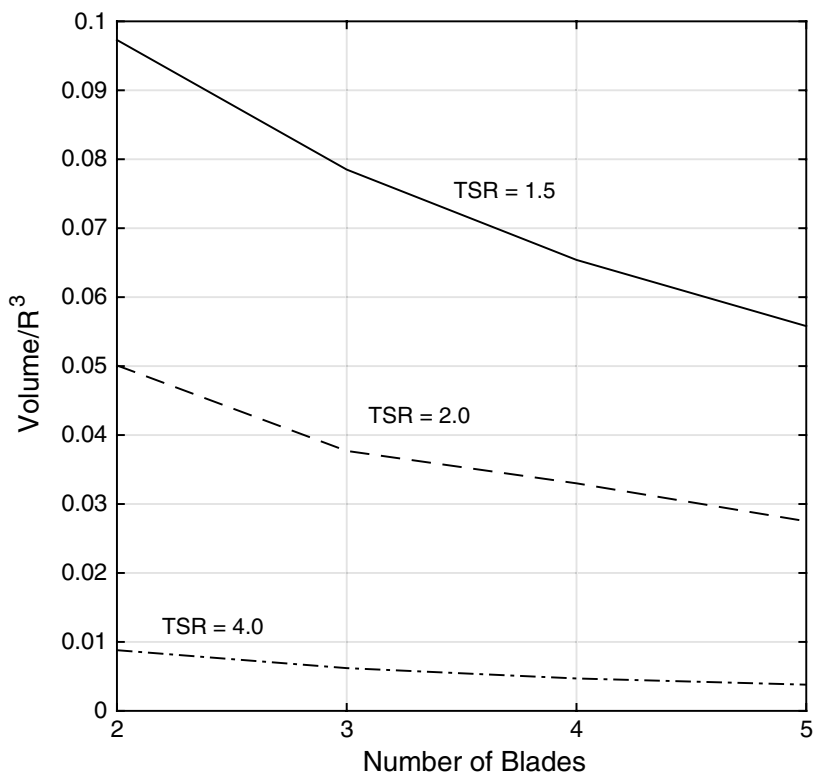

Fig. 12 Dimensionless runner volume (all blades)

the runner, which diminishes with the number of blades. This reduction is more accentuated for low-speed runners and it is very slight for faster runners-with higher values of nominal TSR. For large hydrokinetic turbines (with high TSR), the use of two- or three-blade machines can be 
eventually justified in terms of the blade material weighing, if the performance can be remained the same (as actually is discussed on the use of two-blade machines for offshore wind turbines [21]).

\subsection{Hydrodynamical performance}

The experimental and BEMT results were obtained for the runners with two, three and four blades. Figure 13 presents the power coefficient curves for the different runner configurations, a direct extract from the wind tunnel measurements. The experimental error bars are figured out.

The slight difference in the extract power performance for the three- and four-blade runners can be observed. It is not the case for the two-blade runner, where the levels of the $C_{p}$ curve are clearly lower. The observed experimental uncertainties are compatible with the curves behaviors and other equivalent studies (e.g., [27]). The error bars are greater near the stall points on the blades, where the dynamical effects on the torque can be observed and the variances in the torque measurements are higher.

In Fig. 14, the extrapolation of the experimental results to the prototype scale is considered. This transposition of the results is performed using a Reynolds number correction, as suggested by [28]

$C p_{\text {prot }}=C p_{\text {mod }}\left(\frac{R e_{\text {prot }}}{R e_{\text {mod }}}\right)^{0.12}$

using the parameters presented in Table 3 . The reference radial position $r_{\text {ref }}=\left(R+R_{h}\right) / 2$ is employed to obtain the Reynolds number for the transposition formula, with its respectively chord length.

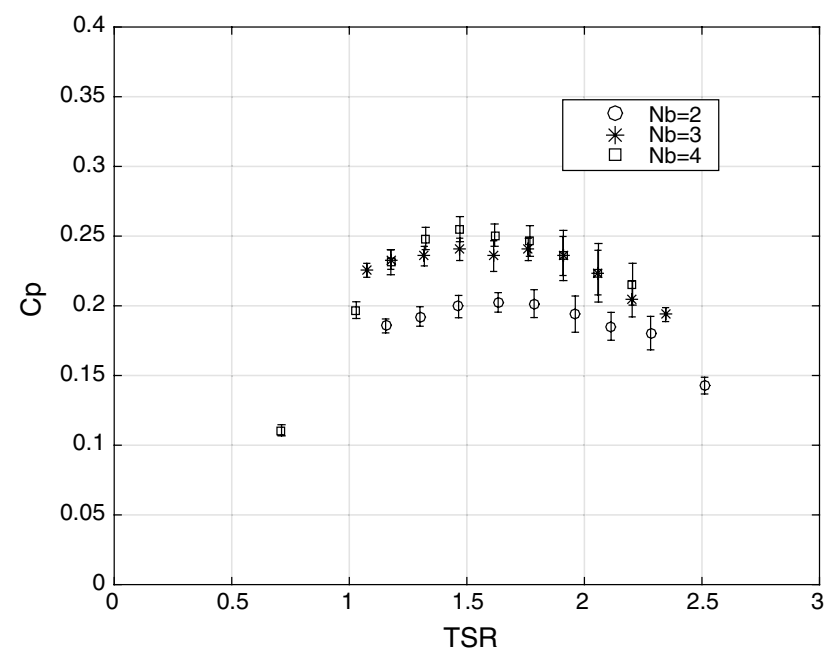

Fig. 13 Power coefficient for wind tunnel experiments-without model-to-prototype extrapolation
Table 3 Model-to-prototype transposition parameters

\begin{tabular}{lll}
\hline Number of blades & Parameter & Value \\
\hline 2 & Chord length (m) & 0.856 \\
& $R e_{\text {prot }}$ & $2.86 \mathrm{e}-06$ \\
& $R e_{\text {model }}$ & $2.86 \mathrm{e}-04$ \\
3 & Chord length (m) & 0.59 \\
& $R e_{\text {prot }}$ & $1.97 \mathrm{e}-06$ \\
& $R e_{\text {model }}$ & $5.88 \mathrm{e}-04$ \\
4 & Chord length (m) & 0.41 \\
& $R e_{\text {prot }}$ & $1.36 \mathrm{e}-06$ \\
& $R e_{\text {model }}$ & $4.08 \mathrm{e}-04$ \\
\hline
\end{tabular}

The experimental results are compared to the numerical simulations of QBLADE, for the different number of blades and for the Reynolds number in the prototype scale.

The adherence of the results is quite well, and it is similar to other reported works involving comparisons between BEMT and wind tunnel experiments-in particular with the use of QBLADE (see Monteiro et. al. [29] for instance) .

Near the design point $(T S R=1.6)$, the experimental and numerical results are very close, which assures the achievement of the blade design methodology. One can observe that the runner with four blades has the higher power coefficient, reaching the maximum value of 0.39 at the target TSR (11\% higher than the three-blade runner), even though the torque coefficient for runners with $N_{b}=3,4$ is very close (considering the experimental results), as shown in Fig. 16. On the other hand, the results for two-blade runner have $C p$ close to 0.3 , poorest than the behavior of the other two runners. A small displacement in the TSR design point to higher values

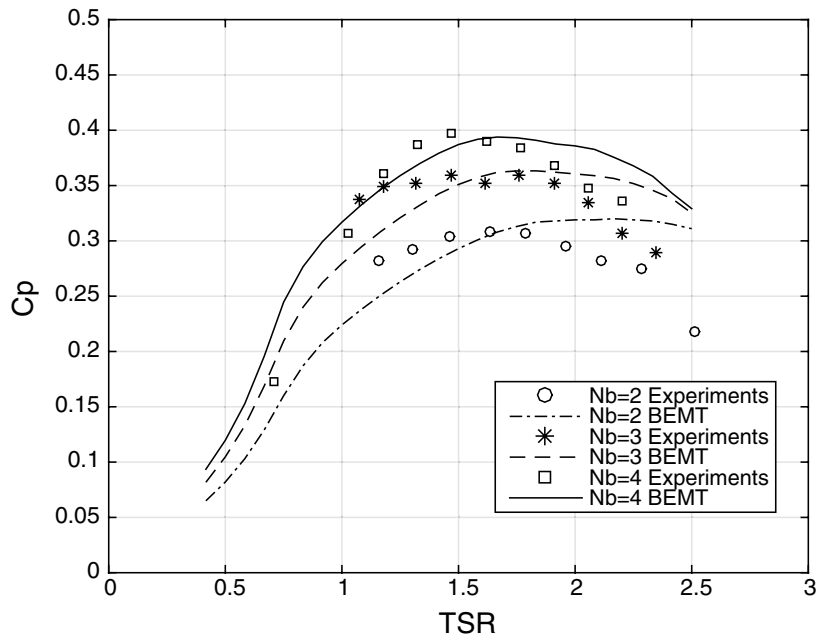

Fig. 14 Power coefficient for BEMT simulations and experimentswith scaling up extrapolation. The error propagation procedure is presented in "Appendix A" 
is observed for runners with a smaller number of blades. It is more clearly observed in the BEMT results.

In order to refine the best results for the four-blade runner, new sets of experiments were performed, obtaining more points in the TSR range. The results are presented in Fig. 15 where the CFD simulations performed in the work of Brasil Junior et. al. [22], for the same runner, are included. The good coherence of all methodologies for the runner performance analysis is observed, assuring a reliable validation of the runner design.

\subsection{Torque coefficient results}

The evaluation of the torque the machine is crucial information to determine the mechanical project to match the runner operation to the electrical generator, selecting also a compatible gearbox and all mechanical requirements for the main shaft.

The torque coefficient is defined by

$C_{M} \equiv \frac{M}{1 / 2 \rho A V_{0}^{2} R}$.

The values of $M$ are directly measured in the turbine model load cell, and in a numerical point of view and in the framework of BEMT, it is computed by the integral of Eq. 26 in all blade sections.

In Fig. 16, the torque coefficient results are plotted as a function of TSR. Both results, experimental and numerical, have shown a typical curve of $C_{M}$, where the high values are

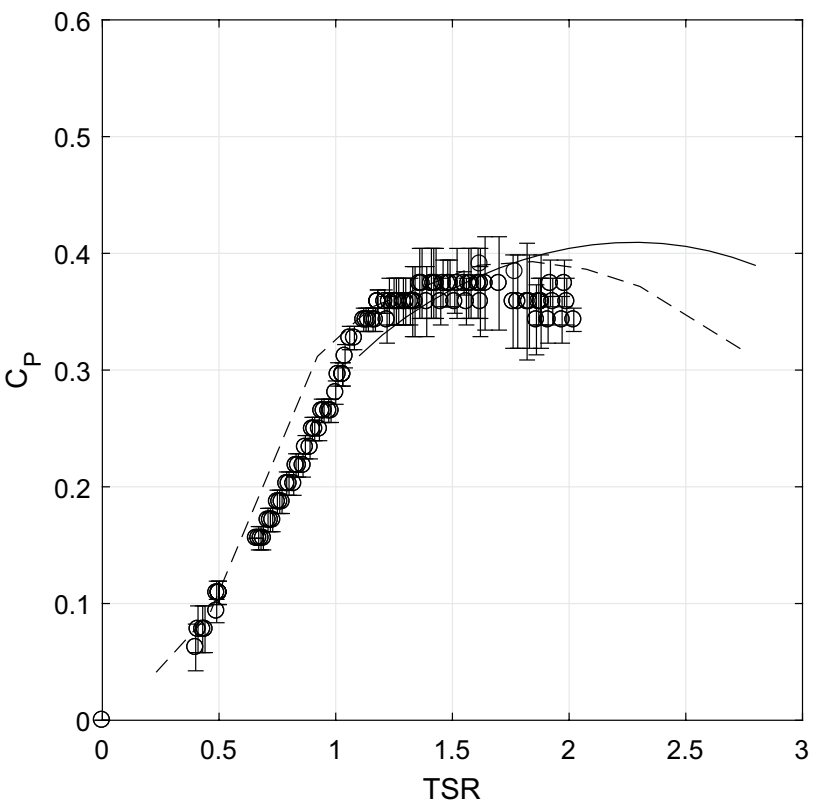

Fig. 15 Power coefficient for four-blade runner. BEMT simulations (-), experiments of Fig. 12 (o) and CFD simulations [22] (- -)

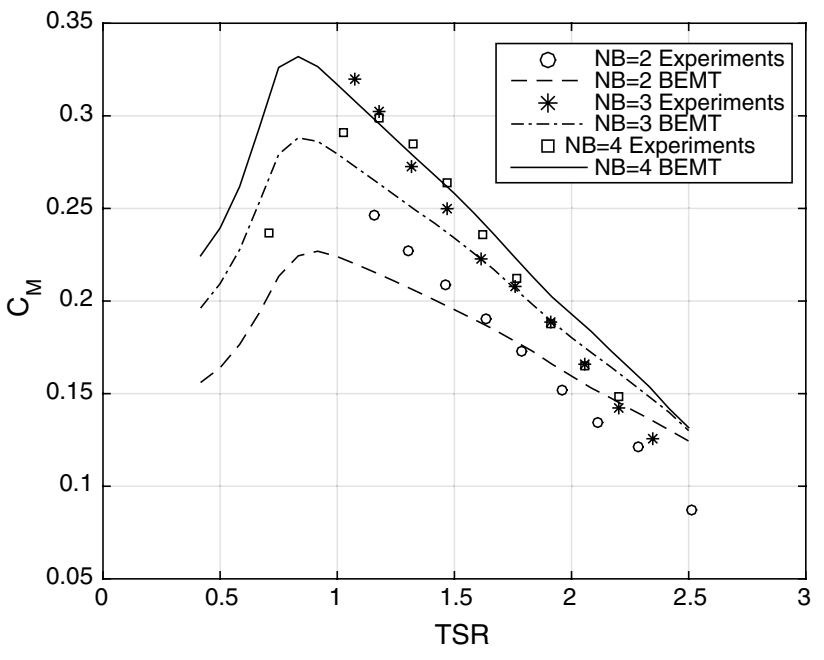

Fig. 16 Torque coefficient for BEMT simulations and experiments

encountered in the regions of low TSRs. It is good in terms of the turbine operation, mainly for the starting condition of the rotating electromechanical set. A needed torque level is required for the low rotation regimes, in order to compensate the friction on the shaft components and powertrain. An important remark is that for $N_{b}=3,4$, a high torque condition is achieved allowing turbines starting even for relatively slow current velocities, which is necessary to the specific requirements of the HK-10 machine.

\section{Conclusions}

A design approach for hydrokinetic axial flow turbines is presented. It was formulated in detail from the basic equations of the blade element moment theory, using the Glauert [7] reasoning for the optimum blade design. In this framework, axial runners for low-rotational-speed propeller hydrokinetic turbine are designed, considering two, three and four blades. The optimum blade shapes have been obtained by the optimized approach described by the cited theories. All runners have firstly evaluated in a qualitative point of view regarding its amount of materials, the feasibility in terms of manufacturing and the limits of the extracted power in the framework of Glauert theory, considering the losses formulations.

The performance of the runners is assessed by means of numerical simulations using BEMT and by means of wind tunnel experiments. In both methodologies, the results have the same behavior and are adherents in terms of tendencies and levels. Those approaches have also been compared to the CFD computations for the four-blade runner, and the agreement of the results allows a good validation or the machine performance, in terms of power coefficient around 0.4 (for 
the four-blade runner). The results showed a tendency of the reduction in $C p$ for runners with two and three blades, comparatively to the runners with more blades.

The torque coefficient results show the same behavior, and an important level of values of $C_{M}$ is observed in the region of low TSRs, in particular for the runners with three and four blades. Those results agree with the classical observations of Glauert and other authors [11,21], more recently.

The results allow good results for the runner design of the turbine HK-10, taking into account its specific characteristics of the low-TSR design point. Considering this requirement, runners with more than three blades are more adequate for the machine performance and also for the torque characteristics, needed in the starting operating regime.

Acknowledgements This work is partially financed by the HYDRO-K project consortium in a context of the ANEEL P\&D Grant, with the partnership of AES-Brasil Company. The authors are grateful to the support of Brazilian Ministry of Education, by means of CAPES, for the PhD scholarships. The France-Brazil cooperation program lying ENSAM-ParisTech and UnB has maintained the international mobility of the researchers.

\section{Propagation of uncertainties}

The experimental uncertainties of this work are given by the sum of instrumental and random errors. For the digital sensors, the instrumental errors are given by the smallest scale value displayed by the instrument and for the analog instruments by the smallest half scale. Random uncertainties were defined as being the standard deviation a set of realizations under the same conditions. In this work, the derived uncertainties can be expressed according to the following primary measurements errors: wind velocity $\left(\delta V_{0}\right)$, rotational speed $(\delta \omega)$ and torque $(\delta T)$. The uncertainty in $C p$ measurements $(\delta C p)$ is computed with the general formula for error propagation [30], as it is given in Eqs. 47, 47 and 48.

$\delta P=\sqrt{\omega^{2} \delta \tau^{2}+\tau^{2} \delta \omega^{2}}$,

$\delta P_{\text {flow }}=\sqrt{\left(3 / 2 \rho A V_{0}^{2} \delta V_{0}\right)^{2}}$,

$\delta C p=\sqrt{\left(P_{\text {flow }}^{-1} \delta P\right)^{2}+\left(P P_{\text {flow }}^{-2} \delta P_{\text {flow }}\right)^{2}}$.

\section{References}

1. Laws ND, Epps BP (2016) Hydrokinetic energy conversion: technology, research, and outlook. Renew Sustain Energy Rev 57:1245-1259

2. Sleiti AK (2017) Tidal power technology review with potential applications in Gulf Stream. Renew Sustain Energy Rev 69:435-441
3. Kumar D, Sarkar S (2016) A review on the technology, performance, design optimization, reliability, techno-economics and environmental impacts of hydrokinetic energy conversion systems. Renew Sustain Energy Rev 58:796-813

4. Yuce MI, Muratoglu A (2015) Hydrokinetic energy conversion systems: A technology status review. Renew Sustain Energy Rev 43:72-82

5. Rehman S, Mahbub Alam M, Alhems LM, Rafique MM (2018) Horizontal axis wind turbine blade design methodologies for efficiency enhancement-a review. Energies 11:506-540

6. Schubel PJ, Crossley RJ (2012) Wind turbine blade design. Energies 5:3425-3449

7. Glauert H (1935) Airplanes propellers. In: Durand WF, Aerodynamic theory Chapter XI. vol. 4, pp. 191-195 (reprinted, Dover, New York, 1963)

8. Burton T, Sharpe D, Jenkins N, Bossanyi E (2001) Wind energy handbook. Wiley, Hoboken

9. Hansen MOL (2015) Aerodynamics of wind turbines, 3rd edn. Routledge, Abingdon

10. Schaffarczyk AP (2014) Introduction to wind turbine aerodynamics. Springer, Berlin

11. Liu S, Janajreh I (2012) Development and application of an improved blade element momentum method model on horizontal axis wind turbines. Int J Energy Environ Eng 3:30

12. Buhl Jr. M L (2005) A new empirical relationship between thrust coefficient and induction factor for the turbulent windmill state. Technical Report NREL/TP-500-36834

13. Benini E (2004) Significance of blade element theory in performance prediction of marine propellers. Ocean Eng 31:957-974

14. Chapman JC, Masters I, Togneri M, Orme JAC (2013) The Buhl correction factor applied to high induction conditions for tidal stream turbines. Renew Energy 60:472-480

15. Goundar JN, Rafiuddin AM (2013) Design of a horizontal axis tidal current turbine. Appl Energy 111:161-174

16. Li W, Zhou H, Liu H, Lin Y, Xu Q (2016) Review on the blade design technologies of tidal current turbine. Renew Sustain Energy Rev 63:414-422

17. Batten WMJ, Bahaj AS, Molland AF, Chaplin JR (2007) Experimentally validated numerical method for the hydrodynamic design of horizontal axis tidal turbines. Ocean Eng 34(7):1013-1029

18. Silva PASF, Shimoya LD, Oliveira TF, Vaz JRP, Amarante Mesquita AL, Brasil Junior ACP (2017) Analysis of cavitation for the optimized design of hydrokinetic turbines using BEM. Appl Energy 185:1281-1291

19. Duquette MM, Swanson J, Visser KD (2003) Solidity and blade number effects on a fixed pitch, $50 \mathrm{~W}$ horizontal axis wind turbine. Wind Eng 27(4):299-316

20. Badshah M, Badshah S, Jan S (2017) Hydrodynamical design of tidal current turbine and the effect of solidity on performance. $\mathrm{J}$ Eng Appl Sci 36:45-54

21. Muhle F, Adaramola MS, Stran L (2016) The effect of the number of blades on wind turbine wake a comparison between 2-and 3-bladed rotors. J Phys Conf Ser 753:032017

22. Brasil Junior ACP, Mendes RCF, Lacroix J, Noguera R, Oliveira TF (2017) Hydrokinetic propeller turbines. How many blades? Am J Hydropower Water Environ Syst 4:16-23

23. Maalawi KY, Badawy MTS (2001) A direct method for evaluating performance of horizontal axis wind turbines. Renew Sustain Energy Rev 5:175-190

24. Drela, M, Giles, M B (1987) A two-dimensional viscous aerodynamic design and analysis code. AIAA-87 0424 report

25. Amarante Mesquita AL, Alves ASG (2000) An improved approach for performance prediction of HAWT using the strip theory. Wind Eng 24:417-430

26. Marten D, Wendler J, Pechlivanoglou J, Nayeri C N, Paschereit C O, (2013) QBLADE: an open source tool for design and 
simulation of horizontal and vertical axis wind turbines. Int J Emerg Technol Adv Eng 3:264-269

27. Kolekar N, Banerjee A (2015) Performance characterization and placement of a marine hydrokinetic turbine in a tidal channel under boundary proximity and blockage effects. Appl Energy 148:121-133

28. Machuca M, Wind tunnel experiments of free flow turbineshydrokinetic and wind turbines (in Portuguese), MSc dissertation in Mechanical Sciences, University of Brasilia, 2016

29. Monteiro JP, Silvestre MR, Piggott H, Andre JC (2013) Wind tunnel testing of a horizontal axis wind turbine rotor and comparison with simulations from two blade element momentum codes. J Wind Eng Ind Aerodyn 123:99-106

30. Taylor JR (1997) An introduction to error analysis. University Science Books, Sausalito, p 327 